\title{
EXHAUSTION FUNCTIONS AND COHOMOLOGY VANISHING THEOREMS FOR OPEN ORBITS ON COMPLEX FLAG MANIFOLDS
}

\author{
Joseph A. Wolf
}

\begin{abstract}
A вsтract. Let $G_{0}$ be a real semisimple Lie group, let $R$ be a parabolic subgroup of the complexification $G$ of $G_{0}$, let $D$ be an open $G_{0}$-orbit in the complex flag manifold $X=G / R$, and let $Y$ be a maximal compact linear subvariety of $D$. First, an explicit parabolic subgroup $Q \subset R \subset G$ is constructed so that the open $G_{0}$-orbits on $W=G / Q$ are measurable and one such orbit $\widetilde{D}=G_{0}(w) \subset W$ maps onto $D$ with affine fibre. Second, it is shown that $D$ is $(s+1)$-complete in the sense of Andreotti and Grauert, $s=\operatorname{dim}_{\mathbb{C}} Y$; thus cohomologies $H^{q}(D ; \mathcal{F})=0$ for $q>s$ whenever $\mathcal{F} \rightarrow D$ is a coherent analytic sheaf. This was known [7] for the case of measurable open orbits, and the proof uses that result on $\widetilde{D}$. Third, it is shown that the space $M_{D}$ of compact linear subvarieties of $D$ is a Stein manifold. For that, a strictly plurisubharmonic exhaustion function is constructed as in the argument [9] for the case of measurable open orbits.
\end{abstract}

\section{Background and statement of results}

Let $G_{0}$ be a connected reductive real Lie group, $\mathfrak{g}_{0}$ its real Lie algebra, and $\mathfrak{g}=\mathfrak{g}_{0} \otimes_{R} \mathbb{C}$ the complexification. As usual, $\operatorname{Int}(\mathfrak{g})$ denotes the complex connected semisimple Lie group of all inner automorphisms of $\mathfrak{g}$, consisting of the $\operatorname{Ad}(g)$ as $g$ runs over any connected Lie group $G$ with Lie algebra $\mathfrak{g}$. Given

$$
\mathfrak{r}: \quad \text { parabolic subalgebra of } \mathfrak{g}
$$

we have the complex flag manifold

$$
X=G / R: \quad \text { all } \operatorname{Int}(\mathfrak{g}) \text {-conjugates of } \mathfrak{r}
$$

where $R$ is the parabolic subgroup of $G$ that is the analytic subgroup for $\mathfrak{r}$.

Received January 11, 1995.

Research partially supported by N.S.F. Grant DMS 9321285. 
$G_{0}$ acts on $X$ through its adjoint action on $\mathfrak{g}$. Since we will only be interested in the $G$-orbits and their structure, we may, and do, assume that

$G$ is connected, simply connected and semisimple, and $G_{0} \subset G$.

The $G_{0}$-orbit structure of $X$ is well understood [8]. There are only finitely many orbits; in particular, there are open orbits. If $x \in X$ let $\mathfrak{r}_{x}$ be the corresponding parabolic subalgebra of $\mathfrak{g}$; that is, if $x=g R$ then $\mathfrak{r}_{x}=\operatorname{Ad}(g) \mathfrak{r}$. Let $\xi \mapsto \bar{\xi}$ denote complex conjugation of $\mathfrak{g}$ over $\mathfrak{g}_{0}$. Then $\mathfrak{r}_{x} \cap \overline{\mathfrak{r}_{x}}$ contains a Cartan subalgebra of $\mathfrak{g}$ of the form $\mathfrak{h}=\mathfrak{h}_{0} \otimes_{\mathbb{R}} \mathbb{C}$ where $\mathfrak{h}_{0}$ is a Cartan subalgebra of $\mathfrak{g}_{0}$. Let $\Delta=\Delta(\mathfrak{g}, \mathfrak{h})$ denote the root system. Fix

$$
\Delta^{+}=\Delta^{+}(\mathfrak{g}, \mathfrak{h}): \text { positive root system }
$$

such that the corresponding ${ }^{1}$ Borel subalgebra

$$
\mathfrak{b}=\mathfrak{h}+\sum_{\alpha \in \Delta^{+}} \mathfrak{g}_{-\alpha} \text { is contained in } \mathfrak{r}_{x} .
$$

Then there is a unique set $\Phi$ of simple roots such that

$$
\mathfrak{r}_{x}=\mathfrak{r}_{x}^{r}+\mathfrak{r}_{x}^{-n}, \quad \mathfrak{r}_{x}^{r}=\mathfrak{h}+\sum_{\alpha \in \Phi^{r}} \mathfrak{g}_{\alpha}, \quad \mathfrak{r}_{x}^{-n}=\sum_{\beta \in \Phi^{n}} \mathfrak{g}_{-\beta}
$$

where

$$
\begin{aligned}
& \Phi^{r} \text { consists of all roots that are linear combinations from } \Phi, \\
& \Phi^{n} \text { consists of all positive roots that are not contained in } \Phi^{r} .
\end{aligned}
$$

Here $\mathfrak{r}_{x}^{-n}$ is the nilradical of $\mathfrak{r}_{x}$ and $\mathfrak{r}_{x}^{r}$ is a reductive complement. Given $\mathfrak{h}$ and $\Delta^{+}(\mathfrak{g}, \mathfrak{h})$, every parabolic subalgebra of $\mathfrak{g}$ is $\operatorname{Int}(\mathfrak{g})$-conjugate to one of the form (1.5), for a unique set $\Phi$ of simple roots.

In the context of (1.4), one knows [8, Theorem 4.5] that

$$
\begin{aligned}
& G_{0}(x) \text { is open in } X \text { if and only if } \\
& \qquad \text { we can choose } \mathfrak{h} \text { and } \Delta^{+} \text {such that } \overline{\Delta^{+}}=-\Delta^{+} .
\end{aligned}
$$

\footnotetext{
${ }^{1}$ Our parabolic subalgebras, which include Borel subalgebras, have nilradicals that are sums of negative root spaces. This is so that holomorphic tangent spaces will be spanned by positive root spaces, so that in turn, positive linear functionals will correspond to positive vector bundles.
} 
Here note that $\overline{\Delta^{+}}=-\Delta^{+}$implies that $\mathfrak{h}_{0}$ contains a regular elliptic element, so that $\mathfrak{h}_{0}$ is a fundamental (as compact as possible) Cartan subalgebra of $\mathfrak{g}_{0}$. Fix

$$
D=G_{0}(x) \subset X \text { : open real group orbit on } X,
$$

$\mathfrak{h}, \Delta^{+}$: Cartan subalgebra and positive root system, and

$K_{0}$ : maximal compact subgroup of $G_{0}$

such that $\mathfrak{k}_{0} \cap \mathfrak{h}$ is a Cartan subalgebra of $\mathfrak{k}_{0}$. Here $\mathfrak{k}_{0}$ and $\mathfrak{k}=\mathfrak{k}_{0} \otimes \mathbb{C}$ are the real and complexified Lie algebras of $K_{0}$. The isotropy subgroup of $G_{0}$ at $x$ is $G_{0} \cap R_{x}$, which has Lie algebra $\mathfrak{g}_{0} \cap \mathfrak{r}_{x}$. Now

$$
D \cong G_{0} /\left(G_{0} \cap R_{x}\right) \text { and } G_{0} \cap R_{x} \text { has complexified Lie algebra } \mathfrak{r}_{x} \cap \overline{\mathfrak{r}_{x}}
$$

Most of the work on open orbits has been done in the case $[8, \S 6]$ of a measurable open orbits - the case where $D$ carries a $G_{0}$-invariant measure. If $D$ is measurable then, in fact, the measure is induced by the volume form of a $G_{0}$-invariant indefinite-kähler metric. The following conditions are equivalent, and $D$ is measurable if and only if they hold [8, Theorem $6.3]$.

$$
\begin{aligned}
& G_{0} \cap R_{x} \text { is the centralizer of a torus subgroup of } K_{0} \cap R_{x}, \\
& \mathfrak{r}_{x} \cap \overline{\mathfrak{r}_{x}} \text { is reductive, } \\
& \mathfrak{r}_{x} \cap \overline{\mathfrak{r}_{x}}=\mathfrak{r}_{x}^{r}, \\
& \overline{\mathfrak{r}_{x}^{n}}=\mathfrak{r}_{x}^{-n} \text { where } \mathfrak{r}_{x}^{n}=\sum_{\beta \in \Phi^{n}} \mathfrak{g}_{\beta} .
\end{aligned}
$$

In general, $D=G_{0}(x)$ is open in $X$ if and only if $\overline{\mathfrak{r}_{x}^{n}} \subset \mathfrak{r}_{x}$, which is implied by the last of the conditions (1.9). For $\mathfrak{r}_{x}^{n}$ represents the holomorphic tangent space to $X$ at $x$, thus to $D$ at $x$ in the case of an open orbit, so in that case $\overline{\mathfrak{r}_{x}^{n}}$ represents the antiholomorphic tangent space.

The conditions (1.9) are automatic if $K_{0}$ contains a Cartan subgroup of $G_{0}$, that is, if $\operatorname{rank} K=\operatorname{rank} G$, in particular if $G_{0} \cap R_{x}$ is compact. They are also automatic if $R$ is a Borel subgroup of $G$. More generally, they are equivalent $[8$, Theorem 6.7$]$ to the condition that $\overline{\mathfrak{r}}$ be $\operatorname{Int}(\mathfrak{g})$-conjugate to the parabolic subalgebra

$$
\mathfrak{r}^{-}=\mathfrak{r}^{r}+\mathfrak{r}^{n} \text { where } \mathfrak{r}^{n}=\sum_{\beta \in \Phi^{n}} \mathfrak{g}_{\beta}
$$

of $\mathfrak{g}$ that is called the opposite of $\mathfrak{r}$.

Compare (1.10) with (1.5): $\mathfrak{r}$ and $\mathfrak{r}^{-}$have the same reductive part, but their nilradicals are "opposite" so that $\mathfrak{g}$ is the vector space direct sum of $\mathfrak{r}^{r}, \mathfrak{r}^{-n}$ and $\mathfrak{r}^{n}$. 
Whether $D$ is measurable or not, $\mathfrak{k} \cap \mathfrak{r}_{x}$ is a parabolic subalgebra of $\mathfrak{k}$, for $\Delta^{+}$consists of all roots whose value on some element $\xi \in \mathfrak{k}_{0} \cap \mathfrak{h}$ has positive imaginary part. It follows that

$$
Y=K_{0}(x) \cong K_{0} /\left(K_{0} \cap R_{x}\right) \cong K /\left(K \cap R_{x}\right)
$$

is a complex submanifold of $D$.

Furthermore, $Y$ is not contained in any compact complex submanifold of $D$ of greater dimension. So $Y$ is a maximal compact subvariety of $D$. We will refer to

$$
M_{D}=\{g Y \mid g \in G \text { and } g Y \subset D\}
$$

as the linear cycle space or the space of maximal compact linear subvarieties of $D$. Since $Y$ is compact and $D$ is open in $X, M_{D}$ is open in

$$
M_{X}=\{g Y \mid g \in G\} \cong G / L
$$

where

$$
L=\{g \in G \mid g Y=Y\} \text {, closed complex subgroup of } G \text {. }
$$

Thus $M_{D}$ has the natural structure of a complex manifold. The point of this paper is to prove

1.14. Theorem. Let $D=G_{0}(x) \subset X$, open orbit on the complex flag $X=G / R$. Let $Y=K_{0}(x)$, maximal compact subvariety, as above. Denote $n=\operatorname{dim}_{\mathbb{C}} D$ and $s=\operatorname{dim}_{\mathbb{C}} Y$. Then $D$ is $(s+1)$-complete in the sense of Andreotti and Grauert [1]: there is an exhaustion function $\phi: D \rightarrow \mathbb{R}$ whose Levi form $\mathcal{L}(\phi)=\sqrt{-1} \partial \bar{\partial} \phi$ has at least $n-s$ eigenvalues $\geqq 0$. In particular, if $\mathcal{F} \rightarrow D$ is a coherent analytic sheaf and $q>s$ then the sheaf cohomology $H^{q}(D ; \mathcal{F})=0$.

Schmid and I proved this theorem some years ago [7] for measurable open orbits.

1.15. Theorem. Let $D$ be an open $G$-orbit on a complex flag manifold $X=G / R$. Then the linear cycle space $M_{D}$ is a Stein manifold.

I proved this a couple of years ago [9] in the case where the open orbit $D$ is measurable. 


\section{The overlying measurable orbit}

In this section we show that an arbitrary open orbit $D=G_{0}(x) \subset X$ is the base of a canonical holomorphic fibration $\pi_{D}: \widetilde{D} \rightarrow D$ where $\widetilde{D}$ is a measurable open $G_{0}$-orbit in a certain flag manifold $W$ that lies over $X$. We then take a close look at that fibration and its relation to the maximal compact linear subvarieties.

Fix the open orbit $D=G_{0}(x) \subset X=G / R$ and consider the parabolic subalgebra $\mathfrak{r}^{-}=\mathfrak{r}^{r}+\mathfrak{r}^{n} \subset \mathfrak{g}$ opposite to $\mathfrak{r}_{x}=\mathfrak{r}=\mathfrak{r}^{r}+\mathfrak{r}^{-n}$. Denote

$$
\mathfrak{q}=\mathfrak{r} \cap \overline{\mathfrak{r}^{-}}
$$

As $D$ is open, so $\mathfrak{r}^{-n} \cap \overline{\mathfrak{r}^{-n}}=0, \mathfrak{q}$ is the sum of a nilpotent ideal $\mathfrak{q}^{-n}$ and a reductive subalgebra $\mathfrak{q}^{r}$ given by

$$
\begin{aligned}
& \mathfrak{q}^{r}=\mathfrak{r}^{r} \cap \overline{\mathfrak{r}^{r}} \text { and } \\
& \qquad \mathfrak{q}^{-n}=\left(\mathfrak{r}^{r} \cap \overline{\mathfrak{r}^{n}}\right)+\left(\mathfrak{r}^{-n} \cap \overline{\mathfrak{r}^{r}}\right)+\left(\mathfrak{r}^{-n} \cap \overline{\mathfrak{r}^{n}}\right)=\left(\mathfrak{r}^{r} \cap \overline{\mathfrak{r}^{n}}\right)+\mathfrak{r}^{-n} .
\end{aligned}
$$

2.2. Lemma. $\mathfrak{q}$ is a parabolic subalgebra of $\mathfrak{g}$, and $\mathfrak{q} \cap \overline{\mathfrak{q}}=\mathfrak{r}^{r} \cap \overline{\mathfrak{r}^{r}}$, which is reductive.

Proof. By construction $\mathfrak{q}$ is the sum of the parabolic subalgebra $\left(\mathfrak{r}^{r} \cap \overline{\mathfrak{r}^{r}}\right)+$ $\left(\mathfrak{r}^{r} \cap \overline{\mathfrak{r}^{n}}\right)$ of $\mathfrak{r}^{r}$ with the nilradical $\mathfrak{r}^{-n}$ of $\mathfrak{r}$. The assertion follows.

Let $Q$ denote the parabolic subgroup of $G$ corresponding to $\mathfrak{q} \subset \mathfrak{g}$ and let $W$ denote the corresponding flag manifold $G / Q$. Our choice of $R$ was such that $\mathfrak{r}=\mathfrak{r}_{x}$ where $x \in X$ and $D=G_{0}(x)$ is the open orbit under study. Let's check that we have implicitly made the corresponding choice on $W$.

2.3. Lemma. Define $w \in W$ by $\mathfrak{q}=\mathfrak{q}_{w}$. Then $\widetilde{D}=G_{0}(w)$ is a measurable open $G_{0}$-orbit on $W$, and $g w \mapsto g x$ defines a surjective holomorphic projection $\pi_{D}: \widetilde{D} \rightarrow D$. Finally, the following are equivalent: (i) $D$ is measurable, (ii) $\widetilde{D}=D$, (iii) $\pi_{D}$ is one to one, and (iv) $Q=R$.

Proof. To see that $\mathfrak{q} \cap \overline{\mathfrak{q}}$ is reductive we compute

$$
\mathfrak{q} \cap \overline{\mathfrak{q}}=\left(\mathfrak{r} \cap \overline{\mathfrak{r}^{-}}\right) \cap\left(\overline{\mathfrak{r}} \cap \mathfrak{r}^{-}\right)=\left(\mathfrak{r} \cap \mathfrak{r}^{-}\right) \cap\left(\overline{\mathfrak{r}} \cap \overline{\mathfrak{r}^{-}}\right)=\mathfrak{r}^{r} \cap \overline{\mathfrak{r}^{r}}=\mathfrak{q}^{r}
$$

Now $G_{0}(w)=\widetilde{D}$ is a measurable open orbit. As $Q \subset R$ we have the natural projection of $W$ onto $X$ :

$$
\pi_{X}: W \rightarrow X \text { by } \pi_{X}(g w)=g x
$$


in other words

$$
\pi_{X}: G / Q \rightarrow G / R \text { by } \pi_{X}(g Q)=g R .
$$

It is holomorphic, and $\pi_{D}$ is the restriction of $\pi_{X}$ to the open orbit $\widetilde{D}$, thus also holomorphic. Further, $\pi_{D}$ is surjective by construction. Finally, the equivalence statement is immediate.

2.4. Lemma. Let $\mathfrak{u}=\left(\mathfrak{r}^{r} \cap \overline{\mathfrak{r}^{-n}}\right)+\left(\mathfrak{r}^{-n} \cap \overline{\mathfrak{r}^{r}}\right)$, nilradical of $\mathfrak{r} \cap \overline{\mathfrak{r}}$, and let $U$ be the corresponding complex analytic subgroup of $G$. Then $U$ is unipotent, $\mathfrak{u}_{0}=\mathfrak{g}_{0} \cap \mathfrak{u}$ is a real form of $\mathfrak{u}, U_{0}=G_{0} \cap U$ is a real form of $U, U(w)=U_{0}(w)$, and $\pi_{D}: \widetilde{D} \rightarrow D$ is a holomorphic fibre bundle with structure group $U$ and affine fibres $\pi_{D}^{-1}(g x)=g U_{0}(w)$. If $g \in G_{0}$ then the holomorphic tangent space to $g U_{0}(w)$ at $g(w)$ is represented by $\operatorname{Ad}(g)\left(\mathfrak{r} \cap \overline{\mathfrak{r}^{-n}}\right)$ and the antiholomorphic tangent space is represented by $\operatorname{Ad}(g)\left(\mathfrak{r} \cap \overline{\mathfrak{r}^{n}}\right)$.

Proof. Here $U$ is the nilradical of $R \cap \bar{R}$ so $U_{0}=G_{0} \cap U$ is the nilradical of the isotropy subgroup $G_{0} \cap R$ and is a real form of $U$. Note $\mathfrak{u}=\mathfrak{v}+\overline{\mathfrak{v}}$ where $\mathfrak{v}=\mathfrak{r}^{r} \cap \overline{\mathfrak{r}^{-n}}=\mathfrak{u} \cap \mathfrak{q}^{n}$, and where $\overline{\mathfrak{v}}=\mathfrak{u} \cap \mathfrak{q}^{-n}$. Both are subalgebras; $\mathfrak{v}$ represents the holomorphic tangent space of $U_{0}(w)$ at $w$ and $\overline{\mathfrak{v}}$ represents the antiholomorphic tangent space. Note $[\mathfrak{v}, \overline{\mathfrak{v}}]=0$.

Now $U(w)=V(w)=U_{0}(w)$ is the fibre over $x$ of $\pi_{D}: \widetilde{D} \rightarrow D$, and $G_{0} \cap R$ is the semidirect product of its unipotent radical $U_{0}$ and a Levy complement $G_{0} \cap Q$. Thus $\pi_{D}: \widetilde{D} \rightarrow D$ satisfies $\pi_{D}^{-1}\left(g \cdot\left(G_{0} \cap R\right)\right)=$ $g U_{0} \cdot\left(G_{0} \cap Q\right)$; in terms of the complex groups this is the same as $g V \cdot Q$. Now we can express $\pi_{D}$ as quotient of $G_{0} /\left(G_{0} \cap Q\right)$ by the action of $U_{0}$ on the right. Thus, the surjective holomorphic map $\pi_{D}$ is the projection of a principle $U_{0}$-bundle. The assertions follow.

2.5. Lemma. Denote $\tilde{Y}=K_{0}(w)$. Then $\tilde{Y}=K(w)$, $\tilde{Y}$ is a maximal compact complex subvariety of $\widetilde{D}$, and $\left.\pi_{D}\right|_{Y}$ is a biholomorphic diffeomorphism of $\widetilde{Y}$ onto $Y$.

Proof. The first two assertions are the analog of (1.11) for $\widetilde{D}$. As $\pi_{D}(k w)=$ $k x$ it is clear that $\pi_{D}(\tilde{Y})=Y$. The restriction $\left.\pi_{D}\right|_{Y}$ is nonsingular by homogeneity, and is injective because the compact affine subvariety $\widetilde{Y} \cap$ $U_{0}(w)$ must be reduced to a point. The third assertion follows.

2.6. Lemma. Let $M_{D}$ denote the linear cycle space of $\widetilde{D}$, as in (1.12). Then $\pi_{D}$ induces a finite covering $\dot{\pi}_{D}: M_{D} \rightarrow M_{D}^{\prime}$ where $M_{D}^{\prime}$ is an open subset of $M_{D}$.

Proof. Let $g \in G$. Then $g \widetilde{Y} \in M_{D}$ if and only if $g K_{0} Q \subset G_{0} Q$. Similarly $g Y \in M_{D}$ if and only if $g K_{0} R \subset G_{0} R$. Note that $g k_{0} Q \subset G_{0} Q$ implies 
$g k_{0} R=g k_{0} Q R \subset G_{0} Q R=G_{0} R$. Thus $g \widetilde{Y} \in M_{D}$ implies $g Y \in M_{D}$. Now $\pi_{D}$ induces a map of $\dot{\pi}_{D}: M_{D} \rightarrow M_{D}$.

Let $M_{D}^{\prime}$ denote the image of $\dot{\pi}_{D}$. Let $L$ and $\widetilde{L}$ denote the respective $G$-stabilizers of $Y$ and $\widetilde{Y}$. Then $[9,(1.4)]$ shows that $\widetilde{L}$ is a subgroup of finite index in $L$, so $\dot{\pi}_{D}$ is the restriction to an open set of the finite cover $G / \widetilde{L} \rightarrow G / L$. Now $M_{D}^{\prime}$ is open in $M_{D}$, and $\dot{\pi}_{D}$ restricts on each topological component of $\dot{\pi}_{D}^{-1}\left(M_{D}^{\prime}\right)$ to a covering of $M_{D}^{\prime}$.

\section{Pushing down the exhaustion function}

Recall the result of [7], which applies to measurable open orbits. It says that the measurable open orbit $\widetilde{D}=G_{0}(w) \subset W$ carries a real analytic exhaustion function $\widetilde{\phi}$, constructed as follows.

Let $Q=Q_{w}$ be given by a set $\Gamma$ of simple roots relative to a Cartan subalgebra $\mathfrak{h}_{0} \subset \mathfrak{g}_{0} \cap \mathfrak{q}$ and a positive root system such that $\overline{\Delta^{+}}=-\Delta^{+}$, in the way that $R=R_{x}$ is described in (1.5) by the set $\Phi$. Here $\Gamma \subset \Phi$.

Let $\lambda=2 \rho_{G / Q^{r}}$, sum of the roots in $\Gamma^{n}$. The corresponding holomorphic line bundles over $\widetilde{D}$ and $W$ are the duals $\mathbb{K}_{D}^{*} \rightarrow \widetilde{D}$ and $\mathbb{K}_{W}^{*} \rightarrow W$ of the canonical line bundles. As $e^{\lambda}: H_{0} \rightarrow \mathbb{C}^{\times}$is unitary we have

$$
h_{0}: \quad G_{0} \text {-invariant hermitian metric on } \mathbb{K}_{D}^{*} \rightarrow \widetilde{D},
$$

and $\mathbb{K}_{\tilde{D}}^{*} \rightarrow \widetilde{D}$ has curvature form

$$
\omega_{0}=2 \pi \sqrt{-1} d_{0} \lambda=-\partial \bar{\partial} \log h_{0}
$$

where $d_{0}$ refers to Lie algebra cohomology of $\mathfrak{g}_{0}$. The maximal compact subgroup $K_{0} \subset G_{0}$ is the fixed point set of a Cartan involution $\theta$ of $G_{0}$. Here we may assume that $\theta\left(H_{0}\right)=H_{0}$. On the Lie algebra level, $\mathfrak{g}_{0}=\mathfrak{k}_{0}+\mathfrak{p}_{0}$ where $\mathfrak{p}_{0}$ is the $(-1)$-eigenspace of $d \theta$, and $\mathfrak{g}_{u}=\mathfrak{k}_{0}+\sqrt{-1} \mathfrak{p}_{0}$ is the Lie algebra of a compact real form $G_{u}$ of $G$. $e^{\lambda}$ remains a well defined unitary character on the Cartan subgroup $H_{u}$ of $G_{u}$ with Lie algebra $\mathfrak{h}_{u}=\left(\mathfrak{h}_{0} \cap\right.$ $\left.\mathfrak{k}_{0}\right)+\sqrt{-1}\left(\mathfrak{h}_{0} \cap \mathfrak{p}_{0}\right)$. Thus we have

$$
h_{u}: \quad G_{u} \text {-invariant hermitian metric on } \mathbb{K}_{W}^{*} \rightarrow W,
$$

and $\mathbb{K}_{W}^{*} \rightarrow W$ has curvature form

$$
\omega_{u}=2 \pi \sqrt{-1} d_{u} \lambda=-\partial \bar{\partial} \log h_{u} .
$$

If $\xi$ and $\eta$ are holomorphic tangent vectors to $W$ at $w$, say

$$
\xi=\sum_{\alpha \in \Gamma^{n}} \xi_{\alpha} \in \mathfrak{q}^{n} \text { and } \eta=\sum_{\alpha \in \Gamma^{n}} \eta_{\alpha} \in \mathfrak{q}^{n} \text { where } \xi_{\alpha}, \eta_{\alpha} \in \mathfrak{g}_{\alpha},
$$


then

$$
\begin{aligned}
& \sqrt{-1} \partial \bar{\partial} \log h_{0}(\xi, \eta)=\pi \sum_{\alpha, \beta \in \Gamma^{n}}(\lambda, \alpha)\left\langle\xi_{\alpha}, \overline{\eta_{\beta}}\right\rangle \text { and } \\
& \sqrt{-1} \partial \bar{\partial} \log h_{u}(\xi, \eta)=\pi \sum_{\alpha, \beta \in \Gamma^{n}}(\lambda, \alpha)\left\langle\xi_{\alpha}, \overline{\overline{\eta_{\beta}}}\right\rangle
\end{aligned}
$$

where $\overline{\eta_{\beta}}$ refers to complex conjugation of $\mathfrak{g}$ over $\mathfrak{g}_{0}$ and $\overline{\overline{\eta_{\beta}}}$ refers to conjugation over $\mathfrak{g}_{u}$. Here $\overline{\bar{\lambda}}=-\lambda$ and $\overline{\Gamma^{n}}=-\Gamma^{n}=\overline{\overline{\Gamma^{n}}}$. The parabolic subalgebra $\mathfrak{q}$ is $\theta$-stable, in particular $\theta \mathfrak{q}^{n}=\mathfrak{q}^{n}$, so $\mathfrak{q}^{n}=\left(\mathfrak{q}^{n} \cap \mathfrak{k}\right)+\left(\mathfrak{q}^{n} \cap \mathfrak{p}\right)$.

The hermitian form $\sqrt{-1} \partial \bar{\partial} \log h_{0}$ is negative definite on $\mathfrak{q}^{n} \cap \mathfrak{k}$ and positive definite on $\mathfrak{q}^{n} \cap \mathfrak{p}$, while $\sqrt{-1} \partial \bar{\partial} \log h_{u}$ is negative definite on all of $\mathfrak{q}^{n}$. More precisely,

$$
\begin{gathered}
\sqrt{-1} \partial \bar{\partial} \log h_{0}(\xi, \eta)=\sqrt{-1} \partial \bar{\partial} \log h_{u}(\xi, \eta)\left(\xi, \eta \in \mathfrak{q}^{n} \cap \mathfrak{k}, \overline{\bar{\eta}}=\bar{\eta}\right), \\
\sqrt{-1} \partial \bar{\partial} \log h_{0}(\xi, \eta)=\sqrt{-1} \partial \bar{\partial} \log h_{u}(\xi, \eta)\left(\xi, \eta \in \mathfrak{q}^{n} \cap \mathfrak{p}, \overline{\bar{\eta}}=-\bar{\eta}\right),
\end{gathered}
$$

and

$$
\sqrt{-1} \partial \bar{\partial} \log h_{0}\left(\mathfrak{q}^{n} \cap \mathfrak{k}, \mathfrak{q}^{n} \cap \mathfrak{p}\right)=0=\sqrt{-1} \partial \bar{\partial} \log h_{u}\left(\mathfrak{q}^{n} \cap \mathfrak{k}, \mathfrak{q}^{n} \cap \mathfrak{p}\right) .
$$

This last uses the hermitian property of $\sqrt{-1} \partial \bar{\partial} \log h_{0}$ and of $\sqrt{-1} \partial \bar{\partial} \log h_{u}$. We now recall the argument of ([6], [7]) for

3.5. Lemma. Define $\widetilde{\phi}: \widetilde{D} \rightarrow \mathbb{R}$ by $\widetilde{\phi}=\log \left(h_{0} / h_{u}\right)$. Then its Levi form

$$
\mathcal{L}(\widetilde{\phi})=\sqrt{-1} \partial \bar{\partial} \widetilde{\phi}=\sqrt{-1} \partial \bar{\partial} \log h_{0}-\sqrt{-1} \partial \bar{\partial} \log h_{u}
$$

is positive semi-definite with at least $\tilde{n}-\tilde{s}$ eigenvalues $>0$, where $\tilde{n}=$ $\operatorname{dim}_{C} \widetilde{D}$ and $\tilde{s}=\operatorname{dim}_{C} \widetilde{Y}$. On the holomorphic tangent space to $\widetilde{D}$ at $w$ it is positive semi-definite, zero on $\mathfrak{r}^{n} \cap \mathfrak{k}$ and positive definite on $\mathfrak{r}^{n} \cap \mathfrak{p}$. More generally, if $g \in G_{0}$ then $\mathcal{L}(\widetilde{\phi})$ is positive definite along the subspace of the holomorphic tangent space that corresponds to $\operatorname{Ad}(g)\left(\mathfrak{r}^{n} \cap \mathfrak{p}\right)$.

Proof. The assertions at $w$ are the content of (3.4). The holomorphic tangent space to $\widetilde{D}$ at $g(w)$ is represented by $\operatorname{Ad}(g) \mathfrak{r}^{n}$ for any element $g \in G$ of the complex group such that $g(w) \in \widetilde{D}$. Now let $z \in \widetilde{D}$, say with $g_{0}(w)=z=g_{u}(w)$ where $g_{0} \in G_{0}$ and $g_{u} \in G_{u}$. Let $\xi$ and $\eta$ belong to the holomorphic tangent space of $\widetilde{D}$ at $z$ and represent

$$
\operatorname{Ad}\left(g_{0}\right) \xi_{0}=\xi=\operatorname{Ad}\left(g_{u}\right) \xi_{u} \text { and } \operatorname{Ad}\left(g_{0}\right) \eta_{0}=\eta=\operatorname{Ad}\left(g_{u}\right) \eta_{u}
$$


where $\xi_{0}, \xi_{u}, \eta_{0}, \eta_{u} \in \mathfrak{r}^{n}$. Then the invariance properties of $h_{0}$ and $h_{u}$ say that

$$
\begin{aligned}
\sqrt{-1} \partial \bar{\partial} \log h_{0}(\xi, \eta) & =\sqrt{-1} \partial \bar{\partial} \log h_{0}\left(\xi_{0}, \eta_{0}\right) \text { and } \\
& \sqrt{-1} \partial \bar{\partial} \log h_{u}(\xi, \eta)=\sqrt{-1} \partial \bar{\partial} \log h_{u}\left(\xi_{u}, \eta_{u}\right) .
\end{aligned}
$$

Now $\sqrt{-1} \partial \bar{\partial} \log h_{0}$ is positive-definite on the $\tilde{n}-\tilde{s}$-dimensional subspace $\operatorname{Ad}\left(g_{0}\right)\left(\mathfrak{q}^{n} \cap \mathfrak{p}\right)$ of the holomorphic tangent space $\operatorname{Ad}\left(g_{0}\right) \mathfrak{q}^{n}$ at $z$, transversal to the homomorphic tangent space $\operatorname{Ad}\left(g_{0}\right)\left(\mathfrak{q}^{n} \cap \mathfrak{k}\right)$ to $g_{0} Y$ at $z$, and $\sqrt{-1} \partial \bar{\partial} \log h_{u}$ is negative-definite on the entire holomorphic tangent space at $z$. So the difference, which is $\mathcal{L}(\widetilde{\phi})$, is at least positive definite on that transversal.

The next step is to push $\widetilde{\phi}$ down to a smooth exhaustion function on $D$.

3.7. Lemma. If $g \in G_{0}$ then $\left.\sqrt{-1} \partial \bar{\partial} \log h_{0}\right|_{g U_{0}(w)}=0$.

Proof. The holomorphic tangent space $\mathfrak{u} \cap \mathfrak{q}^{n}=\mathfrak{r}^{r} \cap \overline{\mathfrak{r}^{-n}}$ to $U_{0}(w)$ at $w$ has basis given by elements $\xi_{\alpha} \in \mathfrak{g}_{\alpha}$ as $\alpha$ runs over $\Gamma^{n}=\Phi^{r} \cap \overline{-\Phi^{n}}$. Let $\alpha, \beta \in$ $\Gamma^{n}$. If $\overline{\xi_{\beta}} \in \mathfrak{g}_{-\alpha}$ then $\alpha \in \overline{\Phi^{r}} \cap \Phi^{n}$, so then $\alpha \in \Phi^{r} \cap \overline{-\Phi^{n}} \cap \overline{\Phi^{r}} \cap \Phi^{n} \subset \Gamma^{r} \cap \Gamma^{n}$, which is empty. Now use $(3.3 \mathrm{~b})$ to see $\sqrt{-1} \partial \bar{\partial} \log h_{0}\left(\xi_{\alpha}, \xi_{\beta}\right)=0$. Take linear combinations to conclude that $\left.\sqrt{-1} \partial \bar{\partial} \log h_{0}\right|_{U_{0}(w)}$ is identically zero at $w$. As $\sqrt{-1} \partial \bar{\partial} \log h_{0}$ is $G_{0}$-invariant, $\left.\sqrt{-1} \partial \bar{\partial} \log h_{0}\right|_{g U_{0}(w)}$ is identically zero at $g w$, for every $g \in G_{0}$. That proves our assertion.

3.8. Lemma. If $g \in G_{0}$ then $\left.\mathcal{L}(\widetilde{\phi})\right|_{g U_{0}(w)}$ is positive definite.

This shows in particular that the fibres $g U_{0}(w)$ of $\pi_{D}: \widetilde{D} \rightarrow D$ are Stein manifolds. We already know that, because we knew, from unipotence of $U$, that those fibres are affine varieties.

Proof. Lemma 3.7 gives that $\left.\sqrt{-1} \partial \bar{\partial} \log h_{0}\right|_{g U_{0}(w)}$ is identically zero. Since $\sqrt{-1} \partial \bar{\partial} \log h_{u}$ is negative definite, so is $\left.\sqrt{-1} \partial \bar{\partial} \log h_{u}\right|_{g U_{0}(w)}$. Hence, the difference $\left.\mathcal{L}(\widetilde{\phi})\right|_{g U_{0}(w)}=\left.\sqrt{-1} \partial \bar{\partial} \log h_{0}\right|_{g U_{0}(w)}-\sqrt{-1} \partial \bar{\partial} \log h_{u}$ is positive definite.

3.9. Proposition. If $g \in G_{0}$ then $\left.\widetilde{\phi}\right|_{g U_{0}(w)}$ has a unique minimum point $m(g)$, so the function $\phi: D \rightarrow \mathbb{R}$ given by

$$
\phi(g(x))=\widetilde{\phi}(m(g))=\min \left\{\widetilde{\phi}\left(w^{\prime}\right) \mid w^{\prime} \in \pi_{D}^{-1}(g(x))\right\}
$$

is well defined. Also, $\phi$ is a real analytic exhaustion function on $D$.

Proof. Let $g \in G_{0}$. If $c>0$ then $\widetilde{D}_{c}=\left\{w^{\prime} \in \widetilde{D} \mid \widetilde{\phi}\left(w^{\prime}\right) \leqq c\right\}$ is compact because $\widetilde{\phi}$ is an exhaustion function. Thus $\widetilde{D} \cap g U_{0}(w)$ is compact. In 
particular $\left.\widetilde{\phi}\right|_{g U_{0}(w)}$ has an absolute minimum. Let $w_{1} \neq w_{2}$ be relative minima of $\left.\widetilde{\phi}\right|_{g U_{0}(w)}$. Choose a smooth curve $s$ in $g U_{0}(w)$ from $w_{1}$ to $w_{2}$, say $s(0)=w_{1}$ and $s(1)=w_{2}$, with $s^{\prime}(t) \neq 0$ for $0<t<1$. Set $f(t)=$ $d \widetilde{\phi}\left(s^{\prime}(t)\right)=\frac{d}{d t} \widetilde{\phi}(s(t))$. Then $f$ has a relative maximum at some $t_{0}$ between 0 and 1 . Here we use $w_{1} \neq w_{2}$. But Lemma 3.8 says $f^{\prime \prime}(t)>0$ for $0<t<1$. Thus $w_{1}=w_{2}$. We have proved that $\widetilde{\phi}_{g U_{0}(w)}$ has a unique minimum point $m(g) \in g U_{0}(w)$.

Now $\phi: D \rightarrow \mathbb{R}$ is well defined as in (3.10). By construction, $\pi_{D}\left(\widetilde{D}_{c}\right)=$ $D_{c}$, so $D_{c}$ is compact, for every real number $c>0$. Thus $\phi: D \rightarrow \mathbb{R}$ is an exhaustion function. It remains to show that $\phi$ is $C^{\omega}$.

Let $M=\left\{m(g) \mid g \in G_{0}\right\}$, the minimum locus just described. Define $\psi: G_{0} \times U_{0} \rightarrow \mathbb{R}$ by $\psi(g, u)=\widetilde{\phi}(g u(w))$. Then $M$ is the image under $\psi$ of the $C^{\omega}$ subvariety of $G_{0} \times U_{0}$ defined by $d_{\mathfrak{u}_{0}} \psi=0$. Thus $M$ is a $C^{\omega}$ subvariety of $\widetilde{D}$. As $\left.\phi \cdot \pi_{D}\right|_{M}=\left.\widetilde{\phi}\right|_{M}$ now $\phi$ is $C^{\omega}$.

3.11. Remark. The first part of the argument of Proposition 3.9 shows that $m(g)$ is the unique critical point of $\left.\widetilde{\phi}\right|_{g U_{0}(w)}$. The second part of the argument shows that the minimum locus $M=\left\{m(g) \mid g \in G_{0}\right\}$ is a $C^{\omega}$ subvariety of $\widetilde{D}$.

\section{The Levi form of the exhaustion function}

Define $\zeta=\phi \cdot \pi_{D}$, so $\zeta: \widetilde{D} \rightarrow \mathbb{R}$ by $\zeta(g(w))=\widetilde{\phi}(m(g))=\phi\left(\pi_{D}(g(w))\right.$. Then the holomorphic tangent spaces of the fibres of $\pi_{D}$ are in the kernel of the Levi form $\mathcal{L}(\zeta)$, and if $g \in G_{0}$ then $\mathcal{L}(\zeta)_{g(w)}$ has the same number of positive eigenvalues as $\mathcal{L}(\phi)_{g(x)}$. This will allow us to calculate that number.

We start with a simple remark from linear algebra, whose proof is included only because several people have questioned this point.

4.1. Lemma. Let $E$ be a finite dimensional vector space over a real division algebra $\mathbb{F}=\mathbb{R}, \mathbb{C}$ or $\mathbb{H}$. Let $\langle\cdot, \cdot\rangle$ be an hermitian form on $E$, say with "signature" $(p, q, z)$, that is, with $p$ signs,$+ q$ signs - , and $z$ signs 0 . Let $E^{+}$be a positive definite subspace, $m=\operatorname{dim}_{\mathbb{F}} E^{+}$, and let $E^{\prime}$ be a complementary subspace of $E$. Suppose that $\langle\cdot, \cdot\rangle$ has signature $\left(p^{\prime}, q^{\prime}, z^{\prime}\right)$ on $E^{\prime}$. Then $p^{\prime} \geqq p-m$.

Proof. We can divide out the kernel of $\langle\cdot, \cdot\rangle$ and assume $z=0$. Let $E^{++}$be a positive definite subspace of $E$ of dimension $p-m$ and orthogonal to $E^{+}$, so $E^{+} \oplus E^{++}$is positive definite and its orthocomplement $F$ is negative definite. If $\left\{e_{1}, \ldots, e_{m}\right\},\left\{e_{m+1}, \ldots, e_{p}\right\}$ and $\left\{f_{1}, \ldots, f_{q}\right\}$ are respective orthonormal bases of $E^{+}, E^{++}$and $F$, then $E^{\prime}$ has a basis of the form 
$\left\{e_{1}^{\prime}, \ldots, e_{p-m}^{\prime} ; f_{1}^{\prime}, \ldots, f_{q}^{\prime}\right\}$ with $e_{i}^{\prime}=e_{m+i}+u_{i}$ and $f_{i}^{\prime}=f_{i}+v_{i}$ where $u_{i}, v_{i} \in E^{+}$.

Case 1 . $E^{++}=0$. Then $m=p$ and the assertion reduces to $p^{\prime} \geqq 0$.

Case 2. $E^{+}=0$. Then $m=0$ and $p^{\prime}=p$, so the assertion reduces to $p \geqq p$. Case 3. $E^{++} \neq 0 \neq E^{+}$. Making an orthonormal changes of basis in $E^{+}$we may assume that $u_{1}$ is a (possibly zero) multiple of $e_{1}$, say $e_{1}^{\prime}=e_{m+1}+a e_{1}$. Look in $\left\{e_{1}, e_{m+1}\right\}^{\perp}$, with $E^{+}$replaced by $\operatorname{Span}\left\{e_{2}, \ldots, e_{m}\right\}, E^{++}$replaced by $\operatorname{Span}\left\{e_{m+2}, \ldots, e_{p}\right\}$, and $F$ replaced by $\operatorname{Span}\left\{e_{2}^{\prime \prime}, \ldots, e_{p-m}^{\prime \prime} ; f_{1}^{\prime}, \ldots, f_{q}^{\prime}\right\}$, where $e_{i}^{\prime \prime}=e_{m+i}+u_{i}^{\prime \prime}$ and $u_{i}^{\prime \prime}$ is the orthogonal projection of the element $u_{i} \in \operatorname{Span}\left\{e_{1}, \ldots, e_{m}\right\}$ onto $\operatorname{Span}\left\{e_{2}, \ldots, e_{m}\right\}$. By induction on $n$ we have $p^{\prime}-1 \geqq(p-2)-(m-1)$, so $p^{\prime} \geqq p-m$.

Denote complex dimensions of our spaces by

$$
n=\operatorname{dim}_{\mathbb{C}} D, \quad \tilde{n}=\operatorname{dim}_{\mathbb{C}} \widetilde{D}, \quad s=\operatorname{dim}_{\mathbb{C}} Y, \quad \tilde{s}=\operatorname{dim}_{\mathbb{C}} \tilde{Y}
$$

where $Y=K_{0}(x) \subset D$ and $\widetilde{Y}=K_{0}(w) \subset \widetilde{D}$ are the maximal compact subvarieties. Lemma 2.5 implies $s=\tilde{s}$.

4.3. Lemma. Recall the minimum locus $M \subset \widetilde{D}$ of Proposition 3.9 and Remark 3.11. Let $m \in M$ and let $T_{m}^{(1,0)}(M)$ denote the part of the holomorphic tangent space to $\widetilde{D}$ tangent to $M$ at $m$. Then $\left.\mathcal{L}(\widetilde{\phi})\right|_{T_{m}^{(1,0)}(M)}$ has at least $n-s$ eigenvalues $>0$.

Proof. Proposition 3.5 says that $\mathcal{L}(\widetilde{\phi})$ has at least $\tilde{n}-\tilde{s}$ eigenvalues $>0$ at $m$, and $\operatorname{dim}_{\mathbb{C}} \pi_{D}^{-1} \pi_{D}(m)=\tilde{n}-n$. Lemma 4.1 now says that $\left.\mathcal{L}(\widetilde{\phi})\right|_{T_{m}^{(1,0)}(M)}$ has at least $n-\tilde{s}=n-s$ eigenvalues $>0$.

4.4. Lemma. Let $\zeta=\phi \cdot \pi_{D}$ as defined at the start of $\S 4$. Then $\mathcal{L}(\zeta)$ has at least $n-s$ eigenvalues $>0$ at every point of $\widetilde{D}$.

Proof. If $m \in M$ then $\left.\mathcal{L}(\zeta)\right|_{T_{m}^{(1,0)}(M)}=\left.\mathcal{L}(\widetilde{\phi})\right|_{T_{m}^{(1,0)}(M)}$, by construction of $\zeta$. The assertion now follows from Lemma 4.3 .

Proof of Theorem 1.14. As was described earlier, the holomorphic tangent spaces of the $\pi_{D}^{-1}(g(x))$ are in the kernel of $\mathcal{L}(\zeta)$, so $\mathcal{L}(\phi)_{g(x)}$ has the same number of positive eigenvalues as $\mathcal{L}(\zeta)_{g(w)}$. Lemma 4.4 says that this number is $\geqq n-s$. Combining this with Proposition 3.9, we see that $\phi$ is a real analytic exhaustion function on $D$ whose Levi form has at least $n-s$ eigenvalues $>0$ at every point. This completes the proof of Theorem 1.14 . 


\section{The Stein property for $M_{D}$}

Proof of Theorem 1.15. Theorem 1.15 is known [9] when $D$ is measurable, so we assume that it is not measurable. The considerations of $[9, \S 1]$ make no use of measurability. If $M_{X}$ is a projective algebraic variety, then $G_{0} / K_{0}$ is an hermitian symmetric space, $G_{0}$ has a compact Cartan subgroup, and $D$ is measurable. Thus [9, Corollary 1.5] $M_{X}$ is an affine algebraic variety. We have set things up so that the argument of $[9, \S 3]$, for $D$ measurable and $M_{X}$ affine, goes through: push $\phi: D \rightarrow \mathbb{R}^{+}$down to $\phi_{M}: M_{D} \rightarrow \mathbb{R}^{+}$ by $\phi_{M}(g Y)=\sup _{y \in Y} \phi(g(y))$; then $\phi_{M}$ is a $C^{\omega}$ plurisubharmonic function on $M_{D}$ that blows up on the boundary of $M_{D}$ in $M_{X}$. Choose a $C^{\omega}$ strictly plurisubharmonic exhaustion function $N$ on the affine variety $M_{X}$. Then $\phi_{M}+\left.N\right|_{M_{D}}$ is a $C^{\omega}$ strictly plurisubharmonic exhaustion function $M_{D}$, so $M_{D}$ is Stein.

This argument relies on a certain amount of Lie structure theory. D. Barlet pointed out that it could also follow from the general result that the space $\mathcal{C}_{s}(Z)$ of compact complex analytic cycles of pure dimension $s$, in an $(s+1)$-complete complex analytic space $Z$ of finite dimension, is a Stein variety. For that result see [4], which extends results from [2], [3] and [5]. Now observe that $M_{D}=M_{X} \cap \mathcal{C}_{s}(D)$. If one proves that this intersection is a closed subvariety of the Stein variety $\mathcal{C}_{s}(D)$, then the restriction to $M_{D}$ of a strictly plurisubharmonic exhaustion function on $\mathcal{C}_{s}(D)$ will be a strictly plurisubharmonic exhaustion function on $M_{D}$, giving an alternate proof that $M_{D}$ is Stein.

\section{Acknowledgement}

The author thanks the Université de Metz and the Universidad Nacional de Córdoba for hospitality during the preparation of this paper.

\section{References.}

1. A. Andreotti and H. Grauert, Théorèmes de finitude pour la cohomologie des espaces complexes, Bull. Soc. Math. France 90 (1962), 193-259.

2. A. Andreotti and F. Norguet, Problème de Levi et convexité holomorphe pour les classes de cohomologie, Ann. Scuola Norm. Sup. Pisa 20 (1966), 197-242.

3. A. Andreotti and F. Norguet, La convexité holomorphe dans l'espace analytique des cycles d'une variété algébrique, Ann. Scuola Norm. Sup. Pisa 21 (1967), 31-82.

4. D. Barlet, Convexité de l'espace des cycles, Bull. Soc. Math. de France 106 (1978), 373-397. 
5. F. Norguet and Y. T. Siu, Holomorphic convexity of spaces of cycles, Bull. Soc. Math. de France 105 (1977), 191-223.

6. W. Schmid, Homogeneous complex manifolds and representations of semisimple Lie groups, thesis, University of California at Berkeley, 1967.

7. W. Schmid and J. A. Wolf, A vanishing theorem for open orbits on complex flag manifolds, Proc. Amer. Math. Soc. 92 (1984), 461-464.

8. J. A. Wolf, The action of a real semisimple group on a complex flag manifold, I: Orbit structure and holomorphic arc components, Bull. AMS 75 (1969), 1121-1237.

9. J. A. Wolf, The Stein condition for cycle spaces of open orbits on complex flag manifolds, Annals of Math. 136 (1992), 541-555.

Department of Mathematics, University of California, Berkeley, CaliFORNIA 94720

E-mail address: jawolf@math.berkeley.edu 